\title{
56 Gb/s Direct Modulation of an InP-on-Si DFB Laser Diode
}

\author{
A. Abbasi ${ }^{1, *}$, B. Moeneclaey ${ }^{2}$, J. Verbist ${ }^{1,2}$, X. Yin ${ }^{2}$, J. Bauwelinck ${ }^{2}$, G. Roelkens ${ }^{1}$, G. Morthier ${ }^{1}$ \\ ${ }^{I}$ Photonics Research Group, INTEC, Ghent University-IMEC, 9000 Ghent, Belgium \\ ${ }^{2}$ Ghent University - imec, IDLab, Dep. INTEC, 9000 Ghent, Belgium \\ *amin.abbasi@ugent.be
}

\begin{abstract}
We demonstrate $56 \mathrm{~Gb} / \mathrm{s}$ direct modulation of a high bandwidth heterogeneously integrated InP/Si DFB laser, and transmission over $2 \mathrm{~km}$ of non-zero dispersion shifted single mode fiber. The high bandwidth of the laser is achieved by exploiting the photon-photon resonance effect.
\end{abstract}

\section{Introduction}

The exponential growth of communication systems and web-based multimedia applications such as video streaming and cloud services drives the demand for a low-cost, low-power and high-bandwidth optical transceiver, especially for use in optical interconnections inside and between data-centers. In order to address this demand optical modulation at data rates of $50 \mathrm{~Gb} / \mathrm{s}$ and higher is desirable. Future $400 \mathrm{~Gb}$ Ethernet standards aim at lane speeds of $56 \mathrm{~Gb} / \mathrm{s}$ and the use of NRZ-OOK in short reach links. In view of these standards, direct and external modulation at such bitrates has been demonstrated with traditional, monolithic InP devices [1-2].

Since a few years silicon photonics became recognized as a potential technology for optical interconnects due to the high index contrast and the CMOS compatibility. Thanks to this technology, very dense optical integration as well as co-integration with the driver electronics are possible. As a result of the strong potential, there has been significant progress in III-V-on-silicon transceivers. Heterogeneously integrated high speed photodiodes [3] and electro-absorption modulators (EAM) [4] have been reported based on InP-on-Si circuits. However, using an EAM as the transmitter in short reach links comes with a few disadvantages. For example, the use of EAMs will introduce an extra insertion loss. Directly modulated lasers are providing a less complex, more compact and a cost-effective solution. Using the photon-photon resonance effect in monolithically integrated III-V lasers, a world record modulation bandwidth of $55 \mathrm{GHz}$ has been reported [5]. In this paper, we present a heterogeneously integrated DFB laser on a Si waveguide with an extended modulation bandwidth $(34 \mathrm{GHz})$ using the same photon-photon resonance technique. We manage to achieve $56 \mathrm{~Gb} / \mathrm{s}$ NRZ-OOK direct modulation, and transmission of this signal over $2 \mathrm{~km}$ of non-zero dispersion shifted fiber (NZ-DSF) without any equalization.

\section{Device structure and static characteristics}

The device structure is shown schematically in Fig. 1-a and is similar to our previous devices [6]. The active layer consists of $8 \mathrm{InAlGaAs}$ quantum wells, which provide a high differential gain. By over-etching the side $\mathrm{SiO}_{2}$ on the Si-on-insulator (SOI) waveguide circuit, we managed to reduce the bonding thickness between the III-V epitaxial stack and the $\mathrm{Si}$ waveguide to less than $10 \mathrm{~nm}$ (Fig. 1-b). The thin bonding layer allows obtaining a large coupling coefficient $\left(\sim 200 \mathrm{~cm}^{-1}\right.$ in this case). The large coupling coefficient gives a small cavity loss, which together with the large confinement factor of the laser mode in the active layer $(\sim 10 \%)$ results in a low threshold gain and a large differential gain. These are the essential requirements to obtain a high relaxation oscillation frequency. An external cavity is created by making use of the reflections from the grating couplers. By properly designing the position of these grating couplers the resonance frequency can be designed to extend the modulation bandwidth of the laser.
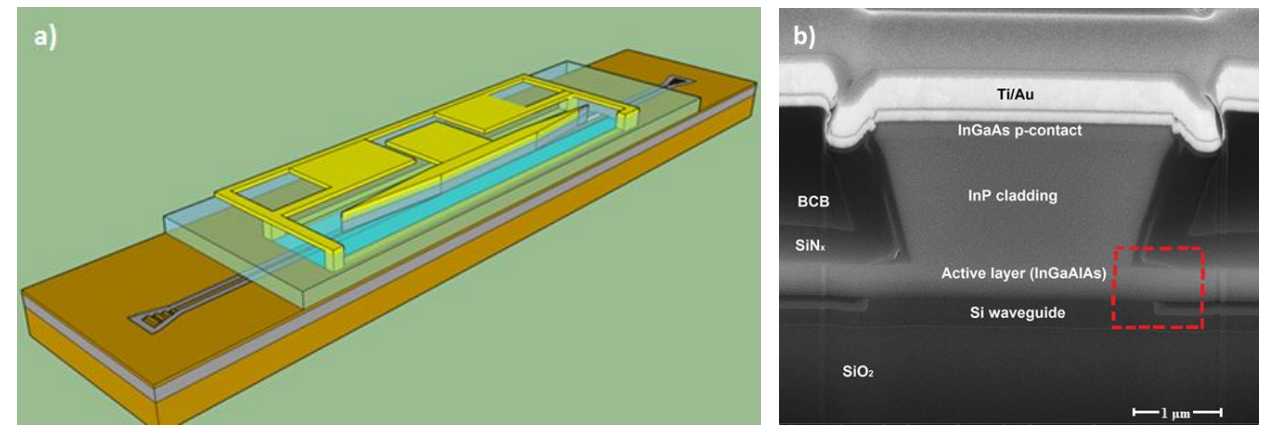

Figure 1. Device structure (a), SEM image of the DFB laser cross-section with an ultra-thin bonding thickness (b). 
The DFB laser has a $6 \Omega$ series resistance, is single mode (emission wavelength of $1570 \mathrm{~nm}$ ) and couples more than $6 \mathrm{~mW}$ output power in the Si rib waveguide for a bias current of $110 \mathrm{~mA}$. The threshold current is $32 \mathrm{~mA}$.

\section{Dynamic characteristics}

First, in order to evaluate the device dynamic properties, small-signal measurements were done with a KEYSIGHT PNA-X $67 \mathrm{GHz}$ network analyzer (Fig. 2). Increasing the laser bias current results in a higher modulation bandwidth. As one can see, there is a low frequency roll off due to the modulation of the tapers, which are used on either side of the DFB laser to couple the light to the Si waveguide and which act as SOAs, and due to spatial hole burning in the laser cavity. When excluding the low-frequency part, we measured a $3 \mathrm{~dB}$ modulation bandwidth of $34 \mathrm{GHz}$ at $110 \mathrm{~mA}$, enabled by a photon-photon resonance at $\sim 32 \mathrm{GHz}$.
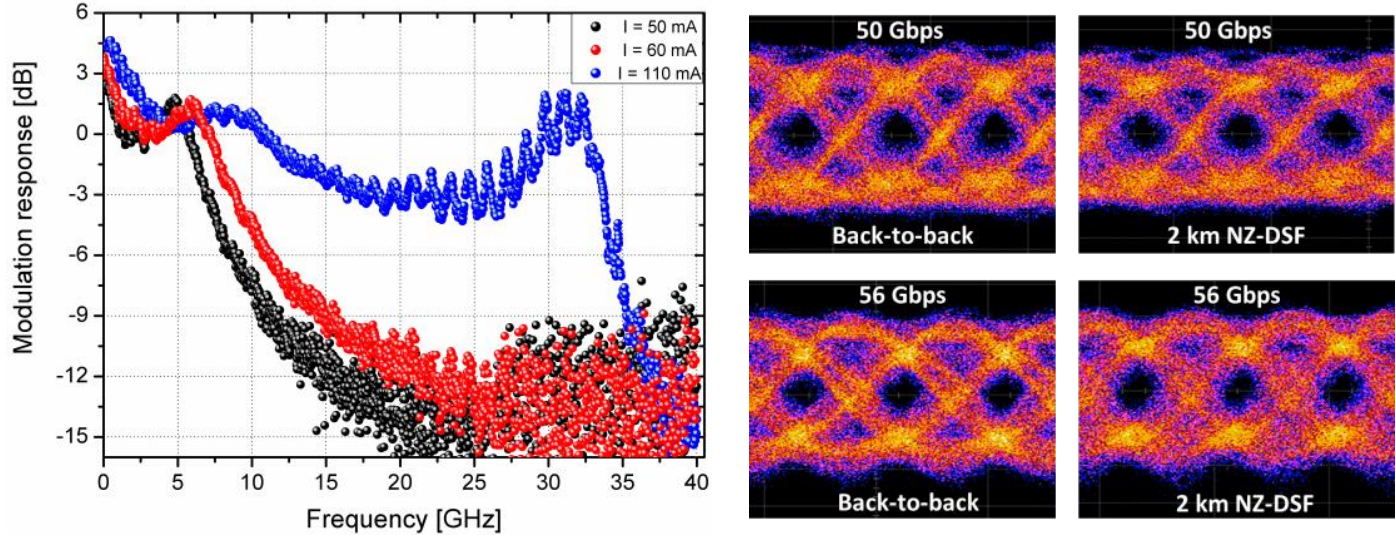

Figure 2. Small signal modulation characteristics for different bias current to the DFB laser, Eye diagrams for 50 and 56 Gbps for the backto-back case (left eyes) and after transmission over $2 \mathrm{~km}$ of NZ-DSF fiber (right eyes).

The large signal measurements were performed using a Keysight M8195A arbitrary waveform generator (AWG) at different bitrates. An RF-voltage of $1 \mathrm{~V}_{\mathrm{pp}}$ from the AWG was applied to the laser to realize large signal modulation while the laser was biased at $110 \mathrm{~mA}$. Using a Root Raised Cosine (RRC) filter with $\alpha=0.7$ at the output of the AWG helps to open the bandwidth limited electrical eye. A pseudo-random bit sequence (PRBS) pattern of $2^{7}-1$ was used in these experiments. An Erbium Doped Fiber Amplifier (EDFA) is used to compensate for the grating coupler loss and to boost the optical signal. An optical filter ( $0.2 \mathrm{~nm}$ bandwidth) is used to suppress the amplified spontaneous emission of the EDFA. Measurements were done both in a back-to-back configuration as well as using a $2 \mathrm{~km}$ span of NZ-DSF fiber with a dispersion of 4.5-6 ps/nm.km at the laser wavelength of $1570 \mathrm{~nm}$. The signal is directly detected by a commercial photodiode and trans-impedance amplifier (TIA) with a bandwidth of $32 \mathrm{GHz}$. The output of the TIA is connected to a real time oscilloscope (Keysight DSA-Z63). Fig. 2 shows the eye diagrams at 50 and $56 \mathrm{Gbps}$ both in back-to-back and $2 \mathrm{~km}$ fiber span configurations. BER measurement results will be presented during the conference, but a BER below the hard-decision FEC threshold was obtained in all cases.

\section{Acknowledgement}

The authors acknowledge 3-5 Lab for supplying the III-V epitaxial wafer. Some of the high speed equipment used in this work has been financed by the Hercules foundation of the Flemish government through project AUGE/13/01.

\section{References}

[1] Theurer, Michael, et al. "2×56 GB/s from a double side electroabsorption modulated DFB laser." Optical Fiber Communications Conference and Exhibition (OFC), 2016. IEEE, (2016).

[2] K. Nakahara, et al, "Direct Modulation at 56 and $50 \mathrm{~Gb} / \mathrm{s}$ of $1.3 \mu \mathrm{m}$ InGaAlAs Ridge-Shaped-BH DFB Lasers", IEEE Phot. Techn. Lett., Vol. 27, No.5, March (2015).

[3] X. Xie et al., "Heterogeneously integrated waveguide-coupled photodiodes on SOI with $12 \mathrm{dBm}$ output power at $40 \mathrm{GHz}$," 2015 Optical Fiber Communications Conference and Exhibition (OFC), Los Angeles, CA, pp. 1-3, (2015).

[4] A. Abbasi, et al, "56 Gb/s Electro-Absorption Modulation of a Heterogeneously Integrated InP-on-Si DFB Laser Diode", accepted for publication in Optical Fiber Communication Conference (OFC), United States, (2017).

[5] Y. Matsui, T. Pham, W. Ling, R. Schatz, G. Carey, H. Daghighian, T. Sudo, and C. Roxlo, "55-GHz Bandwidth Short-Cavity Distributed Reflector Laser and its Application to 112-Gb/s PAM-4," in Optical Fiber Communication Conference Postdeadline Papers, (2016).

[6] A. Abbasi, et al, "High Speed Direct Modulation of a Heterogeneously Integrated InP/SOI DFB Laser", Journal of Lightwave Technology (invited), 34(8), p.1683-1687, (2016). 